ÉGYPTE

monde arabe

\section{Égypte/Monde arabe}

$22 \mid 1995$

Géographies de l'Égypte 1

\title{
Sous les mines, la plage
}

L'espace littoral de la mer Rouge : reconversion et spécialisation d'une interface

\section{Olivier Sanmartin et Jacques Seguin}

\section{(2) OpenEdition}

\section{Journals}

Édition électronique

URL : https://journals.openedition.org/ema/600

DOI : $10.4000 /$ ema. 600

ISSN : 2090-7273

\section{Éditeur}

CEDEJ - Centre d'études et de documentation économiques juridiques et sociales

\section{Édition imprimée}

Date de publication : 30 juin 1995

Pagination : 63-96

ISSN : 1110-5097

\section{Référence électronique}

Olivier Sanmartin et Jacques Seguin, «Sous les mines, la plage », Égypte/Monde arabe [En ligne], 22 I 1995, mis en ligne le 08 juillet 2008, consulté le 07 juillet 2022. URL : http://journals.openedition.org/ ema/600; DOI : https://doi.org/10.4000/ema.600

Ce document a été généré automatiquement le 7 juillet 2022.

Tous droits réservés 


\section{Sous les mines, la plage}

L'espace littoral de la mer Rouge : reconversion et spécialisation d'une interface

Olivier Sanmartin et Jacques Seguin

1 Le premier regard porté sur le territoire de l'Égypte amène tout naturellement à focaliser son attention sur la répartition extraordinaire de la population, sa concentration sur un axe fluvial et ses ramifications. Fortes densités, structure linéaire à petite échelle ${ }^{1}$, l'espace nilotique renvoie à un écoumène longtemps soumis à des contraintes naturelles prégnantes. Source de vie, la vallée est aussi un carcan dont les extensions ne font qu'agrandir un territoire intrinsèquement tourné vers le fleuve. $\mathrm{A}$ cette organisation du territoire est associée une administration centralisée et, en contrepoint, des espaces vides, marginaux, périphériques, lointains. Le désert et les littoraux qui s'y rattachent, enserrant la vallée féconde, sont faiblement maillés, gérés par un tissu lâche de circonscriptions. Les gouvernorats de la mer Rouge et du Sinaï représentent ainsi environ $200.000 \mathrm{~km} 2$, soit un cinquième de la superficie totale de l'Égypte (voir figure 1 page suivante), pour 350.000 habitants, soit $0,6 \%$ de la population égyptienne ${ }^{2}$. 
Figure 1 : Les 26 gouvernorats d'Égypte, d'après le décret n²4 de février 1994

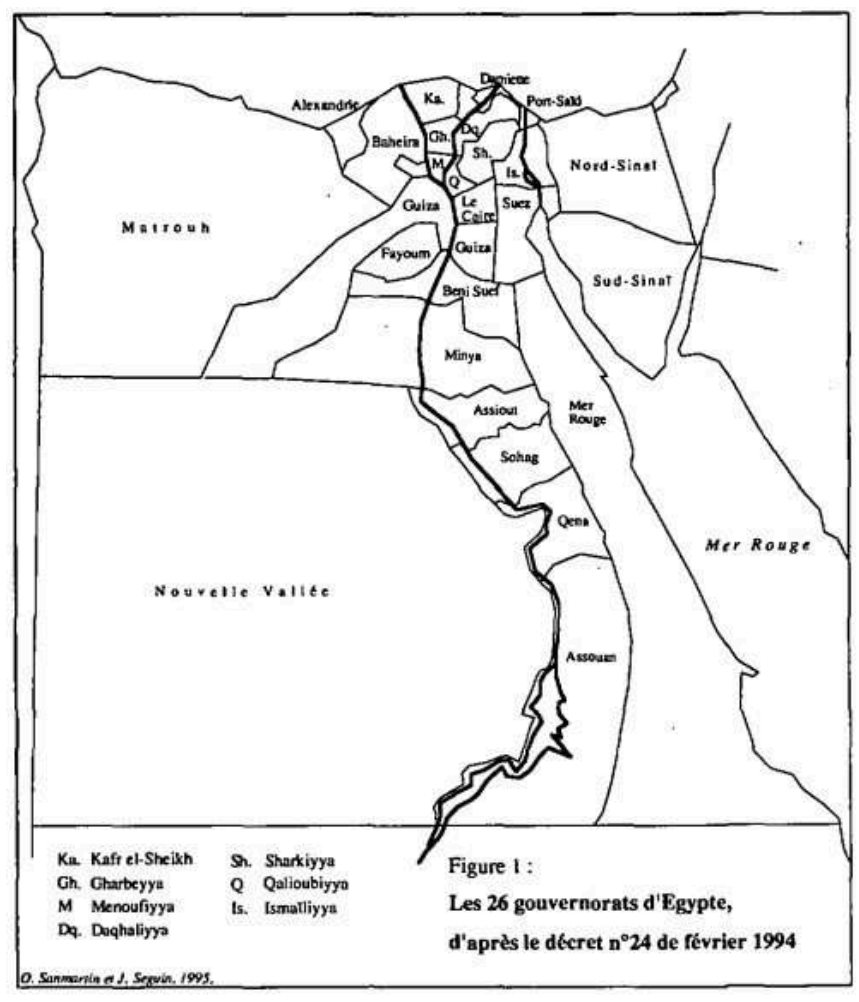

2 Lieux de prélèvement de matières premières, les rivages désertiques et leurs hinterlands ont été longtemps exploités plus qu'intégrés, contrôlés plus qu'administrés. Espaces de captation d'une richesse distribuée vers l'extérieur du pays ou vers la vallée "prédatrice", ce sont des lieux de prélèvement de la rente. Peu peuplés, perçus comme hostiles, ces milieux, marginaux dans l'ossature générale du territoire, n'en ont pas moins été mobilisés par des politiques de contrôle territorial et de sécurité nationale. Dispositifs avancés de protection de la vallée, les littoraux du Sinaï (et leur extension que constitue le canal de Suez), tout comme ceux de la mer Rouge, ont été aménagés pour faire face à la menace, en vue de la confrontation ou de la défense.

Or, depuis la fin des hostilités ouvertes entre l'Égypte et Israël, la fonction, sinon la perception des espaces frontaliers, des dyades orientales, a été modifiée. Avec le mouvement de " conquête du désert ${ }^{3}$ encouragé pour desserrer l'étau démographique sur la vallée, d'une part, et le redéploiement lié à l'occupation des espaces périphériques orientaux "pacifiés ", d'autre part, les zones littorales ont été témoin d'un spectaculaire bouleversement. Organisées, investies par les activités d'un tourisme international en plein développement depuis le milieu des années 80 , leur dynamisme démographique et la croissance spatiale des premiers îlots de peuplement ont modifié leur fonctionnement. Est-ce à dire que ces espaces ont perdu certains attributs de la marginalité ? Leur articulation à l'espace égyptien a-t-elle été modifiée par l'émergence de nouvelles fonctions? Ces changements sont-ils porteurs d'un nouveau mode d'intégration au territoire national? Telles sont les principales questions auxquelles nous tenterons d'apporter des éléments de réponse en mobilisant à la fois une réflexion multi-scalaire et pluridisciplinaire dans une démarche qui privilégiera l'analyse spatiale. 
Le front de l'Est

4 Les côtes de la mer Rouge et le Sinaï, périphériques à l'armature du peuplement égyptien, occupent paradoxalement une situation qui, «s'appréciant par rapport aux voisins et aux lieux en relation, même lointains $»^{4}$, restitue d'autres enjeux à une échelle plus petite. Sur le planisphère, ces rivages sont placés à un carrefour, à un nœud géographique : celui où s'attachent l'Afrique orientale, le Proche-Orient comme marche avancée de l'Asie intérieure, celui où convergent et où finiront par se rejoindre le bassin de la Méditerranée d'un côté et l'océan Indien de l'autre. Isthme maritime, isthme terrestre ${ }^{5}$, ce lieu est éminemment stratégique parce qu'il est le point de rencontre des civilisations et des empires, parce qu'il est axe de circulation, vital pour l'approvisionnement en matières premières, épices ou pétrole. Il est par essence espace de rencontres et de convoitises. Confrontations pour la terre du Sinaï, luttes d'influence pour l'utilisation de la mer Rouge et de ses ports comme point d'ancrage, de positionnement de forces aéronavales pour protéger des ressources et les États riverains qui les possèdent, ces deux éléments de l'équilibre géopolitique régional ont contribué à mettre en avant leurs fonctions stratégiques et militaires.

5 Les points d'accès aux littoraux de la mer Rouge et du Sinaï sont autant de lieux de communication, d'échange et de transfert essentiels. Autant de synapses ${ }^{6}$ dont le contrôle paraît fondamental pour la structuration de l'espace égyptien et, plus largement, de la région proche-orientale.

Le Sinaï, glacis du canal de Suez

6 Espace de circulation, le Sinaï, « porte orientale de l'Égypte », fut et demeure un enjeu pour les États riverains ou des puissances plus lointaines. Qui contrôlait ses routes - au nord, le long du littoral méditerranéen, au centre, en direction du Hedjaz, ou à l'ouest, le long du golfe de Suez - s'assurait le contrôle du commerce oriental par l'isthme et la voie maritime, ou la connexion entre l'Égypte, le Levant et la Péninsule arabique par la voie terrestre.

7 Le percement du canal de Suez, en 1869, scelle le rôle stratégique du Sinaï en le transformant en glacis défensif de la nouvelle voie de navigation. Axe vital des intérêts britanniques, le canal et sa profondeur stratégique que constitue la péninsule seront, à partir de 1882, au centre des préoccupations des occupants. En 1906, ils contraignent l'Empire ottoman au tracé d'une frontière orientale entre Taba, au fond du Golfe d'Aqaba, et Rafah, sur le littoral méditerranéen. Cette ligne, qui confère la responsabilité administrative de la péninsule à l'Égypte et donc à la puissance occupante, inaugure un siècle de présence militaire sur cet espace devenu presqu'île. Ernest Renan, s'adressant à Ferdinand de Lesseps lors du discours de réception de celuici à l'Académie française en 1895, fut pour le moins visionnaire: «Un seul Bosphore avait suffi jusqu'ici aux embarras du monde; vous en avez créé un second, bien plus important que l'autre, car il sert de couloir de communication à toutes les grandes mers du globe. En cas de conflit, il serait le point pour l'occupation duquel tout le monde lutterait de vitesse. Vous avez ainsi marqué la place des grandes batailles de l'avenir. $»^{7}$

8 La structuration de ce désert par la fonction militaire passe alors par le développement d'axes de communication modernes, réseaux de transport et de télécommunication, au premier rang desquels figure le chemin de fer de Palestine. Construit par les Anglais durant la première guerre mondiale, trait d'union entre l'Égypte et le Levant jusqu'en 1948, il assure à l'Empire britannique un contrôle exclusif sur la région. 
9 L'arrivée d'Israël sur la scène internationale et le premier conflit israélo-arabe renforcent la fonction défensive de la péninsule en mettant durablement en sommeil les voies de communication est-ouest. Des aérodromes, des ports, des routes à vocation exclusivement militaire tissent alors un maillage qui exclut le Sinaï de toute intégration au territoire égyptien. Les douanes demeurent à al-Qantara sur le canal de Suez, qui joue alors le rôle de frontière orientale de l'Égypte.

Les espaces de la confrontation israélo-arabe

10 La nationalisation du canal et l'agression tripartite de 1956 donnent lieu à une première occupation du Sinaï par l'armée israélienne. Contraint de se retirer en mars 1957, l'État hébreu n'abandonne pas pour autant ses visées stratégiques sur la péninsule.

11 Alors que les autres espaces désertiques égyptiens, anciens frontier districts hérités de l'administration britannique, accèdent en 1960 au statut de gouvernorat civil, le Sinaï est maintenu dans sa fonction militaire. Hormis quelques lieux de vie liés aux exploitations pétrolières et minières, la région d'al-'Arîsh et les implantations bédouines, le Sinaï reste un no man's land dont Tsahal s'empare sans difficultés lors de l'offensive de juin 1967. Le glacis s'inverse alors et Israël met en place, sur la rive orientale du canal de Suez, une ligne de défense qui se veut imperméable, la ligne BarLev. L'occupation israélienne du Sinaï, entre 1967 et 1982, inaugure un nouveau mode de gestion de l'espace pour la péninsule. Les communications s'ouvrent à l'est, l'exploitation pétrolière s'intensifie - en 1973, Israël tirera du Sinaï jusqu'à $80 \%$ de sa consommation pétrolière ${ }^{8}$ - et une colonisation humaine du territoire est entreprise, destinée au développement agricole au nord et touristique au sud.

L'Égypte, ainsi amputée de son appendice oriental, se voit contrainte de replier sa ligne de défense sur le littoral de la mer Rouge, qui connaissait, depuis le début des années 60, un timide développement touristique. Le minage de la côte, les postes de surveillance et les infrastructures militaires du désert oriental en général, transforment la physionomie d'un espace traditionnellement voué à l'échange. La crainte d'un contournement par les forces israéliennes de la ligne de front située sur le canal, par le sud, et les actions de sabotage menées par les commandos (Asyut, pont de Qena...) montrent combien le nord du désert oriental participe du « champ de bataille » de la guerre d'usure. En 1973, des membres de la tribu bédouine des Ma'aza seront affectés à la surveillance des points d'eau'

13 La traversée du canal de Suez, lors de l'offensive d'octobre 1973, met fin à la guerre d'usure que se livraient Égyptiens et Israéliens depuis 1969, de part et d'autre du canal. Mais la réouverture de l'axe commercial et le lancement d'un processus de paix israéloarabe toujours d'actualité ne remettent pas en cause la fonction militaire du Sinaï, tandis que le littoral du désert oriental se retrouve, d'une certaine façon, en marge des préoccupations stratégiques du moment. Les phases de retrait israéliennes, à partir de 1975, se font sous couvert de l'Onu et le traité de Camp David, signé en 1979, prévoit la création d'une force multinationale d'interposition (MFO), qui se met en place en 1982, sans date butoir. En imposant une démilitarisation de la péninsule et une présence étrangère, Camp David, paradoxalement, rend plus aigu le discours sécuritaire égyptien et n'éloigne pas le spectre d'un nouveau conflit. Le retour de la souveraineté égyptienne - limité - sur le territoire et la question de Taba ${ }^{10}$ illustrent la détermination de l'Égypte à vouloir désormais intégrer coûte que coûte le Sinaï, érigé au rang de symbole national, à l'espace territorial.

Des espaces structurés par les exploitations pétrolières et minières 
Si la longue durée de la confrontation entre l'Égypte et Israël a modelé l'organisation des espaces littoraux et de leurs hinterlands, d'autres fonctions les avaient préalablement structurés, et les infrastructures initiales procédaient autant d'un accès aux ressources que de la mise en place d'un contrôle territorial accru de l'État dans ses marges peu peuplées, où la population bédouine pouvait s'opposer au prélèvement ${ }^{11}$. Le désert oriental égyptien et le Sinaï central présentent des structures montagneuses qui les différencient géomorphologiquement des abords de la vallée. Ils renferment de nombreux gisements dont il faut mentionner l'exploitation ancienne, interrompue à plusieurs reprises : mines d'or du wâdi Hamamat, carrières du mont Claudianus ${ }^{12}$, mines de turquoise et de cuivre de la région de Serabit al-Khadîm dans le Sinaï occidental ${ }^{13}$... L'accès et la maintenance de ces sites, qui ont mobilisé hommes et équipements, sont à l'origine d'un premier canevas de routes et de postes de contrôle ${ }^{14}$, en particulier des axes transversaux du Nil à la mer Rouge, ou des voies d'accès drainant les ressources de chacun des versants du désert oriental vers la vallée ou le littoral. Comme le note G. Alleaume $\mathrm{e}^{15}$,

le mouvement d'exploration minéralogique, entre 1820 et 1830, a une première conséquence immédiatement perceptible : la réintégration, dans l'espace égyptien, des déserts, qui cessent d'apparaître sous la forme d'itinéraires de traversées (de la mer Rouge à la vallée par exemple) pour être parcourus en tous sens et devenir en eux-mêmes et pour eux-mêmes objets d'études et d'observations.

15 Cette réintégration se traduira un siècle plus tard par la mise en place des premiers réseaux et des têtes d'exploitation, embryons urbains, en particulier à Hurghada et à Safaga. Le pétrole est à cet égard exemplaire. Les débuts de l'exploration sur les rivages du golfe de Suez datent de la fin du XIXe siècle. Les premiers forages dans la péninsule de Gemsa remontent à 1886, mais c'est véritablement dans le premier quart du siècle suivant que l'activité prend son essor. En $1922^{16}$, Hurghada est la principale zone de production (6.000 tonnes par jour) dans la concession de l'Anglo-Egyptian Oilfields Company (105 puits en 1934), tandis que des forages d'essai ont lieu sur la rive occidentale du Sinaï, à Abû Derba. A cette époque, $150 \mathrm{~km}$ d'oléoducs sont déjà posés pour alimenter la première raffinerie de Suez. Hurghada est alors une petite cité où se juxtaposent le quartier des ingénieurs européens et les baraquements des ouvriers égyptiens. On y trouve même un cinéma. L'exploitation des mines de phosphates entre Safaga et Qusayr date de la même période. En activité depuis $1908^{17}$, elles occupent 1.912 ouvriers égyptiens et 93 Européens en 1921. Pour l'acheminement vers la côte, des voies de chemin de fer sont posées et des ports aménagés. La cité et le port d'Abû Zaynima sur la rive orientale du golfe de Suez sont voués à l'exploitation du ferromanganèse du gabal Umm Bugma, relié au littoral par une voie ferrée. Dès les années 20, la Sinai Mining Compagny occupe 1.500 ouvriers égyptiens et cadres européens et extrait 100.000 tonnes de minerai par $a^{18}$.

16 Le maillage de l'espace, densifié à partir des années 70 par le développement de l'exploitation des gisements d'hydrocarbures ou d'autres matières premières minérales, se réalise dans une dynamique unidirectionnelle: le prélèvement de la ressource et son transport (voir figure 2 page suivante). Les ports de Safaga, d'Hamrawayn et de Qusayr exportent le phosphate; les cités de Ras Gharîb et de Shukayr, plus au nord, sont les lieux de vie des sociétés pétrolières actives dans le golfe de Suez, région qui représente encore $80 \%$ de la production de pétrole de l'Égypte à l'aube du XXIe siècle. La surveillance d'infrastructures de production (dépôts pétroliers, plates-formes off-shore) et de transport (oléoducs, gazoducs, voies ferrées) 
vitales pour la sécurité économique du pays, relève de préoccupations stratégiques en période de tension militaire. Le golfe de Suez, qui est au cœur de cet ensemble spatial, est juridiquement ${ }^{19}$ " un golfe historique ", c'est-à-dire des eaux intérieures, ou en d'autres termes, comme l'explique Karîm Wissa, « a continuation of the land territory unto the sea $»^{20}$. Si elle n'est pas à l'origine de ces structures, la fonction stratégique demeure présente dans le contrôle territorial nécessaire qu'elles imposent. La protection de ressources essentielles à l'économie égyptienne fait des régions orientales des zones prioritaires dans la définition des objectifs militaires.

Du no man's land à l'espace de peuplement prioritaire

Figure 2 : Zone d'exploitation pétrolière et minière

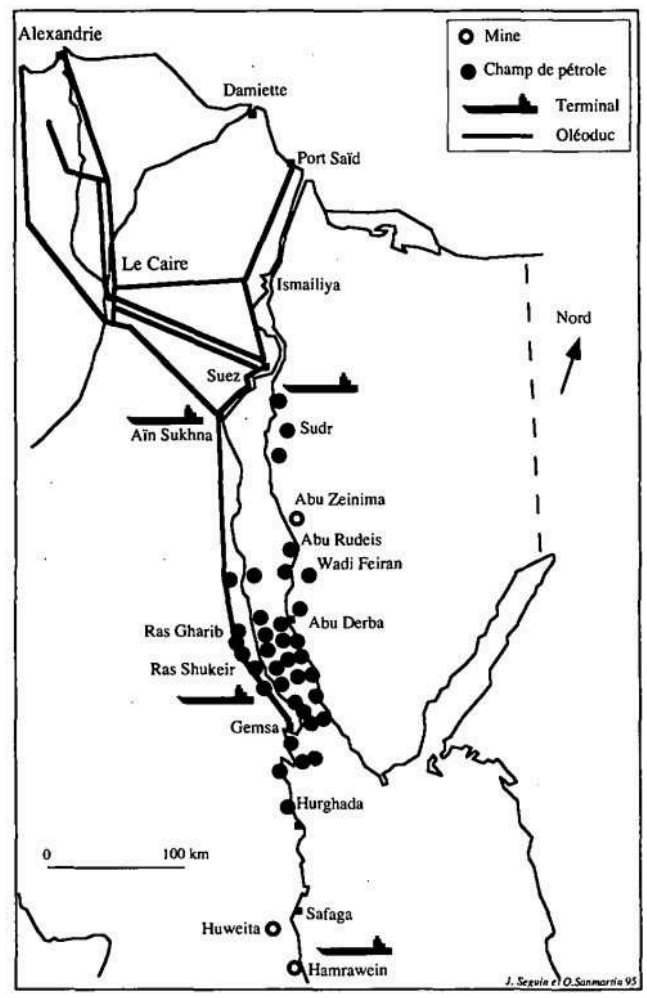

Depuis la fin du conflit, ces espaces, jusqu'alors animés par les seules fonctions de prélèvement et de défense, sont appréhendés dans une nouvelle perspective. Ce n'est pas tant leur perception stratégique qui change, que les modalités de leur mobilisation. Ainsi, à partir de 1973, le gouvernement égyptien semble faire son deuil du no man's land comme stratégie pour le Sinaï. La volonté de peupler le territoire (encore occupé) pour en assurer la sécurité est énoncée par Anouar al-Sadate dans le Manifeste d'octobre 1974: le Sinaï est alors intégré à la "nouvelle carte de l'Égypte » que le président égyptien appelle de ses vœux. La défaite de 1967 avait donné lieu à la prise de conscience, largement répandue, selon laquelle « le Sinaï doit être peuplé d'Égyptiens, car c'est le seul moyen de sauvegarder les frontières orientales de l'Égypte ${ }^{21}$.

Exutoire à l'excédent de population de la vallée autant que barrière humaine à une nouvelle invasion, le Sinaï est devenu un espace de peuplement prioritaire. Plus de vingt ans après la guerre d'octobre, cet objectif ne s'est pas démenti, et les plans à long terme définis pour développer la péninsule proposent tous la mise en valeur du territoire par des millions d'Égyptiens. 
19 Etendue à l'ensemble des littoraux du pays, pour l'essentiel désertiques, cette stratégie se veut mobilisatrice. C'est à un véritable mouvement de conquête du territoire qu'appellent les planificateurs qui, mettant en avant la dégradation des conditions de vie dans la vallée et la surpopulation, produisent un discours d'économie spatiale qui se veut fondateur d'une nouvelle géographie de l'Égypte ${ }^{22}$.

Les éléments d'une redéfinition de la fonction militaire

Si de nouvelles "armes" sont mobilisées pour la sécurité des territoires, les préoccupations stratégiques demeurent. Une redéfinition de la présence militaire s'engage, mais la persistance des représentations conflictuelles et l'instabilité régionale limitent « l'effacement» du rôle, jusqu'alors prépondérant, de la fonction militaire. Ce processus s'opère selon des modalités différentes pour la mer Rouge et le désert oriental, d'un côté, pour le Sinaï, de l'autre.

21 Avec la fin de la confrontation égypto-israélienne, la mer Rouge ne perd pas toute dimension stratégique. Aussi les équipements aéroportuaires et portuaires continuentils d'accueillir des unités militaires. Le port de Safaga, qui abrite des unités de la marine égyptienne, est une échelle pour les bâtiments des flottes occidentales participant à des missions autour de la péninsule arabique, tandis qu'une importante base aéronavale est en activité à $\mathrm{Ra}^{\prime} \mathrm{s} \mathrm{Banas}^{23}$. En dépit de la permanence de cette fonction stratégique à l'échelon régional, le littoral de la mer Rouge a vu disparaître sa fonction de ligne de défense. Des déminages ont été entrepris, des postes de ravitaillement délaissés. Comme l'affirmait récemment le vice-amiral Ahmad 'Ali Fâdil, commandant des forces maritimes égyptiennes, à l'occasion de la fête de ce corps d'armée, «les forces navales sont chargées de combattre la contrebande et le trafic, ainsi que la pollution, le terrorisme et la violation des lois sur la pêche, tout en assurant les différentes opérations de sauvetage maritime le long des côtes égyptiennes et dans les eaux territoriales. $\|^{24}$

Des missions autres, des priorités différentes sont mises en avant : la fonction défensive est remplacée par un dispositif de surveillance des côtes par l'intermédiaire d'une dissémination régulière de postes d'observation. La fin du rôle défensif lié à la confrontation ne signifie pas le retrait total, la démilitarisation, mais elle s'accompagne d'une redéfinition des rôles militaire et stratégique du littoral et de son espace intérieur et, d'une certaine façon, d'une reconfiguration de l'espace investi par les militaires en fonction de la perception de nouvelles menaces.

Le Sinaï, démilitarisé selon une gradation ouest-est imposée par les accords de Camp David, partiellement occupé par une force multinationale, voit se pérenniser une situation de souveraineté limitée sans cesse dénoncée au sein de l'armée comme par les opposants du régime. Pour Sa'd al-Dîn Shazlî, ancien major-général, "c'est une question de fierté : nous avons une force étrangère sur notre sol et nous payons pour la maintenir $»^{25}$. 'Adil Husayn ${ }^{26}$, dans un article qui lui vaudra un mois d'emprisonnement, dénonce la MFO comme étant en réalité le signe d'une présence militaire américaine sur le territoire égyptien ${ }^{27}$.

24 Reprenant à son compte la volonté de statuer sur cette question, le gouvernement égyptien met en avant les coûts de fonctionnement des MFO - qu'il finance pour un tiers, sur un total de 51 millions de dollars pour 1995, à parité avec Israël et les ÉtatsUnis - pour réclamer une réduction des effectifs de la force (actuellement, 2.600 hommes, dont la moitié environ sont américains) et un repli de ses activités sur la 
frontière égypto-israélienne ${ }^{28}$. Une demande en ce sens avait déjà été formulée par l'Égypte en novembre 1992, et rejetée par Israël ${ }^{29}$.

Il s'agit moins, pour Le Caire, d'affaiblir la fonction militaire du Sinaï, perçue comme garantissant la sécurité d'Israël et non celle de l'Égypte, que d'affirmer son droit à en assurer pleinement le contrôle et de proclamer son indépendance à l'égard de la politique étrangère américaine, en arguant d'une conjoncture régionale favorable. Les forces égyptiennes, massivement présentes à l'ouest de Suez, sont prêtes à se déployer dans la péninsule. En effet, le développement économique du Sinaï passe naturellement par l'intégrité territoriale et l'affirmation d'une souveraineté totale, alors que le lancement du processus d'autonomie palestinienne et, surtout, la signature du traité de paix israélo-jordanien ont redéfini les conditions de coexistence régionale par des volets économiques auxquels l'Égypte compte bien être associée.

Le maintien d'un rôle stratégique

De nouvelles menaces, la nécessaire affirmation de la souveraineté, la protection de territoires riches en ressources diverses ou la réactivation permanente d'une mémoire collective toujours centrée sur le conflit égypto-israélien, maintiennent l'Orient égyptien dans un rôle stratégique majeur.

Aux problèmes de souveraineté que pose le Sinaï est venue se greffer la question du triangle de Halaïb, ce territoire situé à la frontière soudanaise, au sud du gouvernorat de la mer Rouge. L'envoi de troupes dans la zone au cours de l'hiver 1992-93, la mise en place d'infrastructures, le recensement et les projets de sédentarisation des populations de la région ${ }^{30}$ signifient, au-delà des intérêts économiques, un repositionnement des forces égyptiennes face à de nouvelles menaces. Le contentieux ${ }^{31}$, rouvert en février 1992 à l'occasion de l'octroi, par le Soudan, d'une concession pétrolière, est un baromètre des relations égypto-soudanaises. Le régime de Khartoum est accusé de prêter assistance aux groupes islamistes armés, en conflit ouvert avec le régime égyptien depuis mars 1992. Après la crise provoquée par l'affaire - ou le mythe - des missiles irakiens (on disait, pendant la guerre du Golfe, qu'ils menaçaient l'Égypte à partir du territoire soudanais), ce nouvel épisode du différend soudanoégyptien illustre la volonté du Caire d'assurer un contrôle territorial total aux avantpostes d'une région en pleine croissance économique, alors que la confrontation armée au centre du pays a durablement sinistré certaines activités, au premier rang desquelles figure le tourisme.

28 En janvier 1993, au plus fort de la crise, le président Moubarak, voulant signifier la détermination de l'Égypte à recouvrer la souveraineté sur la zone contestée, déclarait : «Halaïb, c'est 17.000 fois Taba... » Quant au ministre de l'Agriculture, également vicepremier ministre et secrétaire général du PND (Parti national démocratique au pouvoir), il ajoutait que son pays «ne resterait pas les bras croisés face aux camps d'entraînement de terroristes installés par l'Iran aux frontières de l'Égypte et d'un pays voisin ", et que "les frontières sont plus sûres avec Israël qu'avec le Soudan et la Libye $\aleph^{32}$. Si la crise de janvier 1994, relative à l'arsenal nucléaire israélien et au refus d'Israël d'adhérer au TNP ${ }^{33}$, peut rendre caduque cette dernière analyse, il apparaît clairement que les questions de sécurité restent centrales sur des espaces sous-peuplés, à la merci d'une remise en cause de leur développement.

29 La confrontation avec Israël et la guerre de 1973, à laquelle ont pris part nombre de dirigeants actuels - au premier rang desquels le chef de l'État -, ont relégué au second plan la célébration des événements liés à la révolution de 1952. Une des valeurs 
essentielles du nationalisme demeure le consensus anti-israélien qui continue, semblet-il, de transcender les clivages affectant l'ensemble de la société égyptienne. C'est dans ce contexte que s'opère une mise en scène du Sinaï et de la mer Rouge comme théâtres et enjeux du conflit, mise en scène rythmée par un grand nombre de commémorations qui font de ces espaces des lieux de mémoire sans cesse réactivés. Loin des enjeux centraux du débat politique, la mobilisation de la mémoire collective se perpétue par des référents liés au conflit et à la réintégration des territoires perdus. Fêtes des gouvernorats, des forces armées ou du pétrole se succèdent, réanimant immuablement un même discours consensuel et nationaliste. Le 6 octobre, qui commémore l'offensive victorieuse de l'Égypte en 1973, est la fête des forces armées et fait désormais figure de deuxième fête nationale ; le 21 octobre, fête de la marine, célèbre l'attaque en 1967, au large de Port-Saïd, d'un navire israélien dont le nom, Eilat, est tout un symbole; le 17 novembre est la fête du pétrole qui commémore la récupération par l'Égypte, en 1975, des exploitations pétrolières de la côte occidentale du Sinaï; le 22 janvier, fête du gouvernorat de la mer Rouge, rappelle une attaque israélienne repoussée en 1970 devant l'île de Shadwan au large de Hurghada ; le 19 mars, fête du gouvernorat du SudSinaï, célèbre la récupération de Taba en 1989 ; le 25 avril, fête nationale du Sinaï, commémore la restitution, en 1982, de la péninsule à l'Égypte...

Malgré la pérennisation d'un discours, d'un contexte psychosociologique et de représentations toujours marquées par le conflit avec Israël, des changements sont perceptibles dans la gestion de l'espace. Les acteurs traditionnels semblent passer la main et se cantonner dans un rôle d'observateurs ou d'arbitres, tandis que le secteur privé, présent depuis peu dans la mise en valeur de ces régions, y occupe une place croissante. Cette dialectique du changement voit s'effectuer un transfert de compétences du militaire vers le civil, du public vers le privé, du national vers l'étranger, sur un terrain qui était jusqu'alors chasse gardée de l'État et de son armée. C'est dans ce contexte que s'effectue la conversion vers un secteur aujourd'hui dominant sur ces espaces, le tourisme.

La conversion au tourisme

31 Les hostilités ouvertes, qui faisaient obstacle à l'installation des hommes et au développement de leurs activités dans cet espace - l'investissement par la fonction militaire étant inhibiteur d'autres développements -, ont laissé place à une paix juridiquement fondée. Par un retournement spectaculaire, les littoraux défensifs et militarisés sont devenus le siège d'une des activités les plus attractives qui soit, le tourisme. Embryonnaire à la fin des années 60 , ce secteur va connaître une croissance continue, alimentée en partie par l'infitâh, et va proprement décoller au milieu des années 80 : en vingt ans, le nombre de touristes va passer de 500.000 à 3 millions (voir graphique 1 en annexe).

La nouvelle carte touristique

Le tourisme international a donc investi l'Égypte, dessinant sur le territoire et dans les paysages une nouvelle carte à thème, spécifique. Mais plus que tout autre, ce secteur est sensible aux aléas de la situation politique des pays d'accueil et aux perceptions qui s'y rattachent. La guerre du Golfe avait déjà affecté les mouvements touristiques vers le Moyen-Orient sans épargner la vallée du Nil. A partir de 1992, le développement de la confrontation entre les mouvements islamistes armés et les forces de sécurité - qui s'est traduit, entre autres, par des attentats contre les touristes provoquant la fermeture d'une partie du territoire égyptien - a contribué à accélérer la réorientation 
des flux traditionnellement dirigés vers la vallée. La stratégie du Ministère du Tourisme, qu'explicitent notamment les «zones de développement prioritaires ${ }^{34}$, vient éclairer la tentative de contourner la crise de ce secteur d'activités. En choisissant de sortir de la vallée et de son patrimoine pharaonique comme unique ressource, les planificateurs vont poursuivre deux objectifs : faire naitre des espaces d'attraction aux marges du territoire et diversifier les types de clientèle et de produits en proposant un tourisme récréatif. Comme l'indiquait récemment le ministre du Tourisme, Mamdûh alBaltagi,

le tourisme culturel et historique a cédé la première place au tourisme de divertissement et de distraction en mer Rouge et au Sinaï. (...). Le tourisme vise à la redistribution de la population en Égypte afin de créer une sorte d'harmonie entre l'espace et la population. Le tourisme travaille à créer de nouvelles collectivités urbaines. ${ }^{35}$

Les littoraux du désert oriental, qui ont connu un développement antérieur à la crise, se voient donc réinvestis d'une mission «civilisatrice» à laquelle n'étaient pas prédisposés les premiers investisseurs. Fréquentées depuis les années 60 , les côtes de la mer Rouge, après l'installation de quelques précurseurs comme le Sheraton d'Hurghada, ouvert en 1964 et réouvert en 1979, ou le Club Méditerranée (1977), furent témoin d'un véritable boom de la construction, en particulier à partir de $1982^{36}$. Quant au Sinaï, si ce sont les Israéliens qui ont été les initiateurs du développement touristique durant l'occupation, c'est le règlement de la question de Taba, en 1989, qui a réellement marqué le décollage de l'activité touristique dans la péninsule.

Hors de la vallée, les sites littoraux de la mer Rouge, en prise directe avec l'extérieur du territoire, sont perçus comme des espaces "pionniers": parce qu'ils sont mis en valeur, parce qu'ils induisent un mouvement migratoire, parce qu'ils accueillent des activités très extériorisées, connectées au marché mondial, au sein duquel ils occupent désormais une place reconnue (Sharm al-Shaykh est un centre de plongée de réputation internationale). D'une certaine façon aussi, parce que ces sites font l'objet d'une gestion quasi-exclusive par le secteur privé. En effet, au moment où le discours sur la libéralisation tente de se concrétiser dans divers secteurs de l'économie, le tourisme a sans doute expérimenté plus tôt que les autres une participation active des entrepreneurs. Menée tambour battant par l'ancien ministre du Tourisme, Fu'âd Sultân, la libéralisation est aujourd'hui poussée à un point tel que dans le Sinaï et sur le littoral du désert oriental (soumis en particulier à des problèmes d'approvisionnement en eau), les infrastructures sont prises en charge par le secteur privé. Dans un schéma «classique» d'investissements, il incombe à l'État de mettre à la disposition des usagers les équipements de base, la viabilisation des terrains, le branchement aux réseaux, etc. Parfois, pour alléger les charges publiques, ces opérations peuvent prendre la forme d'un montage en BOT (Build Operate Transfer). Un prestataire de service (en général le pourvoyeur des équipements) assure la construction, gère pendant une quinzaine d'années les installations puis rétrocède l'équipement aux pouvoirs publics au terme de sa concession. En mer Rouge, les contraintes et les coûts (liés à la dispersion et à l'isolement par rapport aux réseaux nationaux) ont conduit dans certains cas à la privatisation totale des équipements, des unités de dessalement et des générateurs fonctionnant pour des sites complètement autonomes. Dans le cas du montage financier de grands projets touristiques, comme à Abû Soma ${ }^{37}$, une société financière achètera le terrain, qu'elle viabilisera grâce à des crédits accordés par la Banque mondiale, puis rétrocédera des lots aux investisseurs potentiels en percevant 
une plus-value. La récente mise en service d'une station d'eau potable à Sharm alShaykh illustre jusqu'à quel point peut être poussée cette logique d'innovation financière : dans ce cas particulier, les agences gouvernementales se sont associées au secteur privé pour la réalisation d'un équipement public ${ }^{38}$.

Les littoraux de la mer Rouge sont ainsi des espaces en phase avec les développements de l'économie-monde, où le rythme de la croissance des activités dépasse celui des services gouvernementaux chargés de l'encadrer, où les initiatives privées relaient non seulement les carences de l'État, mais sont aussi parfois le vecteur d'une certaine forme de modernisation financière et de gestion. La logique ultra-libérale trouve néanmoins ses limites dans une économie marquée depuis plus de quarante ans par un forte présence étatique. Initiateur d'un désengagement total de l'État dans le secteur touristique, l'ancien ministre Fu'âd Sultân semble avoir été écarté en raison de cette politique radicale. C'est à une reprise en main à tout le moins discursive que l'on assiste aujourd'hui, avec le retour d'acteurs traditionnels tels que le Ministère des Travaux publics.

Le dynamisme de ces espaces peut également se mesurer à la forte croissance démographique des agglomérations principales. Hurghada, la capitale du gouvernorat de la mer Rouge, comptait 7.823 habitants en 1976 et plus de 17.000 en 1986, soit une croissance de 9,8\% par an. Safaga, le principal port de ce même gouvernorat, est passé en dix ans de 4.176 à 10.595 habitants. Si, pour le Sinaï, les chiffres sont moins significatifs, le décollage de l'activité touristique étant postérieur au dernier recensement, des localités telles que Tûr (chef-lieu du gouvernorat du Sud-Sinaï), Sharm al-Shaykh, Dahab ou Nuwayba ont connu, entre 1982 et 1986, une croissance moyenne annuelle de $15,5 \%{ }^{39}$.

Les structures d'accueil se sont considérablement développées. En 1979, le parc des infrastructures touristiques de Hurghada ${ }^{40}$ était constitué d'un seul hôtel international, d'un village touristique, d'un guest-house et d'un hôtel de basse catégorie. Quinze ans plus $\operatorname{tard}^{41}$, le site de cette station balnéaire, qui s'étend sur plus de 30 kilomètres, comprend 24 villages touristiques et 18 hôtels. En 1993, le gouvernorat de la mer Rouge disposait de plus de 11.000 lits et les constructions alors entamées équivalaient au double. Sharm al-Shaykh possédait 5 hôtels en 1988 et l'on en dénombrait 36 en 1994 ; la capacité d'accueil de la station a été multipliée par huit entre les deux dates, passant de 565 chambres à plus de $4.000^{42}$. Pour l'ensemble du Sud-Sinaï (4.556 chambres en 1993), la capacité hôtelière devrait être portée à 8.000 d'ici fin 1997, avec un total de 48.000 emplois liés au secteur du tourisme ${ }^{43}$. En 1991, l'ensemble du parc hôtelier du Sinaï et de la mer Rouge, exprimé en nombre de lits, représentait $18,5 \%$ du parc égyptien, et à la fin de 1993, près de $30 \%$.

Tableau 1 : Capacité d'accueil du tourisme égyptien

\begin{tabular}{|l|l|l|l|l|l|l|l|l|l|l|}
\hline 1993 & $\begin{array}{l}\mathrm{H} \text { et } \mathrm{V} \\
(1)\end{array}$ & $\begin{array}{l}\text { Ch. } \\
(2)\end{array}$ & Lits & $\begin{array}{l}\mathrm{H} \text { et } \mathrm{V} \text { en } \\
\text { constr. }\end{array}$ & Ch. & Lits & $\begin{array}{l}\text { Total } \\
(3)\end{array}$ & Ch. & Lits \\
\hline $\begin{array}{l}\text { Nord- } \\
\text { Sinaï }\end{array}$ & 6 & 556 & 1.048 & 4 & & 504 & 1.270 & 10 & 1.060 & 2.318 \\
\hline \hline Sud-Sinaï & 47 & 4.556 & 9.474 & 24 & & 2.780 & 5.658 & 71 & 7.336 & 15.132 \\
\hline
\end{tabular}




\begin{tabular}{|c|c|c|c|c|c|c|c|c|c|}
\hline $\begin{array}{l}\text { Mer } \\
\text { Rouge }\end{array}$ & 43 & 56.956 & 11.307 & 35 & 5.704 & 11.189 & 78 & 11.399 & 22.496 \\
\hline Total & 90 & 10.807 & 21.829 & 63 & 8.988 & 18.117 & 153 & 19.795 & 39.946 \\
\hline Égypte (4) & 504 & 49.024 & 96.886 & 144 & 20.439 & 40.825 & 648 & 69.463 & 13.771 \\
\hline$\%(5)$ & 18 & 22 & 22,5 & 44 & 44 & 44,4 & 24 & 28,5 & 29 \\
\hline
\end{tabular}

(1) Hôtels et villages.

(2) Chambres.

(3) Parc existant et en construction.

(4) Hors Sinaï et mer Rouge.

(5) Sinaï et mer Rouge par rapport au reste du pays.

Source : Tourism in Figures 1993, Ministère du Tourisme.

Les aéroports militaires de Sharm al-Shaykh et de Hurghada ont accédé au statut d'aéroport international et accueillent des vols directs en provenance d'Allemagne ou d'Italie. Alors que le trafic intérieur de l'ensemble des aéroports égyptiens progressait de 13,2 \% entre 1988 et 1993, celui de Sharm al-Shaykh enregistrait une hausse de 370 $\%$, soit 222.000 passagers, et celui d'Hurghada de $140 \%$ (412.000 passagers) ${ }^{44}$.

Les effets d'« insularité »

Les nouveaux espaces touristiques du Sinaï et du désert oriental entretiennent avec la vallée des relations paradoxales, puisque leur intégration à l'économie nationale s'accompagne d'un phénomène de déconnexion spatiale. Bien qu'à l'intérieur du territoire national on se soit affranchi de la distance - de la nécessaire traversée du vide - pour y accéder, le « court-circuitage » de la porte d'entrée aérienne dans le pays - traditionnellement Le Caire - par la mise en service d'aéroports internationaux in situ permettant un accès direct depuis l'Europe, a modifié les caractéristiques de l'espace touristique et de ses relations avec le centre. De plus, la situation du tourisme en Égypte, menacé par le terrorisme, n'est pas étrangère à l'accentuation de l'isolement. Le conflit externe a cédé la place à un conflit interne, et tandis que se dessine un environnement international pacifié, le "front intérieur » semble quant à lui progresser, ou du moins, se déplacer. Il faut ainsi faire face à la «contagion terroriste " qui a gagné le sud (Qéna-Louxor) et pourrait bien s'étendre à l'est: l'attentat de Hurghada, qui a fait trois morts dont un touriste allemand en septembre 1994, «montre que la Jamâ'a islâmiyya pourrait poursuivre sa stratégie de destruction de l'industrie touristique en Égypte, et que ses coups pourraient s'étendre à des régions nouvelles, considérées comme étant à l'abri du terrorisme ${ }^{45}$. La sécurisation de ces nouveaux espaces est donc jugée impérative.

Particulièrement prégnant sur son espace touristique majeur - le golfe d'Aqaba -, l'isolement du Sinaï, déploré par ailleurs, est utilisé pour préserver un secteur en pleine croissance, jusqu'alors épargné par la crise. La structure et la situation de ce " complexe touristique » permettent une déconnexion par rapport au reste du pays. La zone démilitarisée, à l'est du Sinaï, sous contrôle de la MFO et de la police égyptienne, englobe l'ensemble des sites touristiques du golfe. Elle est bornée par un certain nombre de points de contrôle fonctionnant comme autant de "frontières " internes, renforçant « l'insularité » de la région. Les mesures sécuritaires, éminemment contre- 
productives, qui ont cours dans la vallée (escortes, fouilles, présence policière) ne sont pas pratiquées dans le Sinaï. Les contrôles, essentiellement à l'adresse des Égyptiens, ont été renforcés, mais à l'intérieur de la zone touristique, rien ne transparaît... De plus, le Sinaï est un « produit » qui s'adresse essentiellement au marché occidental, et sa promotion est menée de manière spécifique, sans lien avec le tourisme culturel. Loin des zones de turbulences politiques, le tourisme en mer Rouge, essentiellement voué à la pratique de la plongée sous-marine, n'est que rarement associé à la visite des sites pharaoniques ou de la capitale, qui feront éventuellement l'objet d'un autre voyage. Le visa accordé pour le Sinaï est d'ailleurs distinct et ne permet pas d'accéder au reste du territoire.

41 La présence notable des touristes israéliens sur le golfe d'Aqaba constitue également un fort élément de différenciation. Le visiteur qui circule sur la route littorale remarquera la présence des véhicules israéliens, identifiables aux plaques minéralogiques qu'ils conservent exceptionnellement, alors que tout véhicule étranger entrant en Égypte doit être muni d'une immatriculation spéciale. Depuis Camp David en effet, la zone côtière, entre Taba et Sharm al-Shaykh, est accessible aux ressortissants israéliens par la route, sans visa (remplacé par un «droit d'entrée » d'environ 15 \$) et ces derniers constituent aujourd'hui une part importante des «clients» du Sinaï. Le nombre total d'Israéliens se rendant en Égypte et le nombre d'étrangers transitant par le poste frontière de Taba permettent de mesurer l'augmentation constante des flux touristiques entre l'Égypte et Israël.

Tableau 2: Mouvements d'étrangers au poste-frontière de Taba

\begin{tabular}{|l|l|l|}
\hline Années & Entrées & Sorties \\
\hline \hline 1990 & 106.112 & 116.723 \\
\hline \hline 1992 & 160.172 & 167.366 \\
\hline \hline 1993 & 210.946 & 241.150 \\
\hline
\end{tabular}

Tableau 3 : Touristes israéliens en Égypte

\begin{tabular}{|l|l|l|l|l|l|l|l|l|l|}
\hline 1985 & 1986 & 1987 & 1988 & 1989 & 1990 & $1991^{*}$ & 1992 & 1993 & 1994 \\
\hline 24.575 & 32.319 & 71.031 & 62.902 & 122.062 & 75.778 & 96.379 & 110.212 & 123.985 & 213.214 \\
\hline
\end{tabular}

*Janvier à septembre.

Sources : Egypt Tourists Statistics Information 86-90, Egypt: Tourism in Figures, 1990/91 et 1993 (Ministère du Tourisme). Pour 1994 : Le Progrès égyptien, 16/05/95.

Le shekel, la monnaie israélienne, dont le change est limité en Égypte, circule librement dans le sud-est du Sinaï depuis 1982. Cette entorse à la règle est utilisée par les responsables israéliens comme une brèche pour accélérer la normalisation des relations économiques entre les deux pays. A la suite de la visite au Caire du ministre israélien du Commerce en décembre 1994, le ministre égyptien de l'Economie et du 
Commerce extérieur avait d'ailleurs annoncé que «la Banque centrale égyptienne examinait la possibilité d'autoriser la circulation du shekel israélien dans l'ensemble des banques et des bureaux de change $»^{46}$.

Il en va différemment pour le gouvernorat de la mer Rouge dont les flux touristiques sont composites. La " déconnexion » y possède un caractère saisonnier. Si les touristes occidentaux constituent la clientèle essentielle de la région durant la majeure partie de l'année, le phénomène s'inverse au cours de la saison estivale, où les Égyptiens deviennent majoritaires. Les équipements touristiques se sont d'ailleurs diversifiés dans ce sens, puisqu'il existe désormais un parc immobilier de résidences secondaires, notamment à Hurghada, et que la formule du time-sharing, fort peu développée ailleurs, $y$ connaît un grand succès ${ }^{47}$. (Voir graphique 2 en annexe)

44 La déconnexion spatiale, somme toute classique, d'une dialectique d'intégration économique du tourisme international est ici renforcée, nous l'avons souligné, par la conjoncture sécuritaire qui prévaut. La redéfinition de l'économie touristique égyptienne dans les visions à long terme des planificateurs se veut toutefois intégratrice : d'une part, à l'échelle nationale, en tentant de mettre sur pied des circuits associant "nature» et "culture», paysages de la mer Rouge et patrimoine pharaonique, et en projetant un développement plurisectoriel pour les régions dites " reculées »; d'autre part, à l'échelle internationale, par la volonté de créer un espace touristique régional associant, dans un premier temps, Égypte, Israël et Jordanie.

Les projections régionales

Les projets d'aménagement touristique s'orientent dans deux directions qui les inscrivent résolument dans un cadre transnational. Un front de développement progresse vers le sud du gouvernorat de la mer Rouge, tandis qu'un espace touristique multinational se profite sur le golfe d'Aqaba. Mises en relation, ces deux régions doivent contribuer à l'émergence d'une zone de libre échange touristique centrée sur la mer Rouge, dont les modalités ont été ébauchées lors de la conférence de Casablanca en octobre 1994.

Le « front pionnier»

Le front ou plutôt l'espace pionnier entendu comme « la limite atteinte par la mise en valeur, avancée extrême des colons, des migrants, qui viennent établir une colonie dans des terres jusque-là vides ou peu peuplées $\aleph^{48}$ est la catégorie la plus apte à décrire le processus de conquête des littoraux du désert oriental. Presque exclusivement tourné vers la côte, le gouvernorat de la mer Rouge, qui s'est vu amputé de près de la moitié de son territoire en 1994 au profit des gouvernorats de la vallée ${ }^{49}$ - environ $130.000 \mathrm{~km}^{2}$ aujourd'hui contre 203.000 auparavant -, offre sur plus de 400 kilomètres, de Hurghada à Bérénice, des ressources touristiques encore peu exploitées et une formidable réserve foncière. Des secteurs prioritaires y ont été définis, alternant zones de développement et espaces préservés (buffer zone), le confirmant ainsi dans sa qualité pionnière qui, comme le précise Roger Brunet, est « rarement linéaire, mais procède souvent par sauts au long d'axes privilégiés laissant à l'arrière des poches non transformées $»^{50}$.

47 Le développement des infrastructures procède de cette logique de croissance réticulaire. Après Hurghada, c'est Bérénice qui devrait être dotée d'un aéroport international tandis que de nouvelles liaisons transversales devraient permettre, entre autres, de relier la ville à Assouan. D'autres axes transversaux, maritimes cette fois, doivent améliorer les relations avec l'Arabie Saoudite et le golfe d'Aqaba ${ }^{51}$. 
La « Riviera » de la mer Rouge

Apparu de longue date dans le discours des décideurs, notamment israéliens, le projet d'une « Riviera " proche-orientale qui associerait dans un même ensemble Taba (et plus largement la côte orientale du Sinaï), Eilat et Aqaba, s'est brusquement concrétisé à la suite de l'accord de paix israélo-jordanien et de l'ouverture d'un poste frontière sur le wâdi 'Araba. Lors de la cérémonie de signature du traité, le premier ministre israélien, appelant à "faire une oasis fertile de ce qui n'est aujourd'hui qu'un champ de mines ", insista sur l'importance que revêt pour Israël le volet touristique, par la circulation des hommes et des biens et la nécessaire sécurisation des espaces qu'il implique dans la mise en place d'un hypothétique « marché commun proche-oriental ».

Séparées pendant près de cinquante ans, Aqaba et Eilat ont connu une forte croissance qui fait aujourd'hui des deux villes un ensemble urbain, touristique et industriel unique dans cette partie de la mer Rouge. Avec 35.000 habitants $^{52}$, Eilat, devenue zone franche en $1985^{53}$, est un des centres touristiques les plus importants d'Israël (2 millions de nuitées en 1994) et le port qui relie l'État hébreu à l'Extrême-Orient. A quelques kilomètres, au-delà d'une frontière jusqu'alors imperméable, Aqaba, le seul port de Jordanie, compte plus de 40.000 habitants.

Figures 3 : Axes et infrastructures touristiques

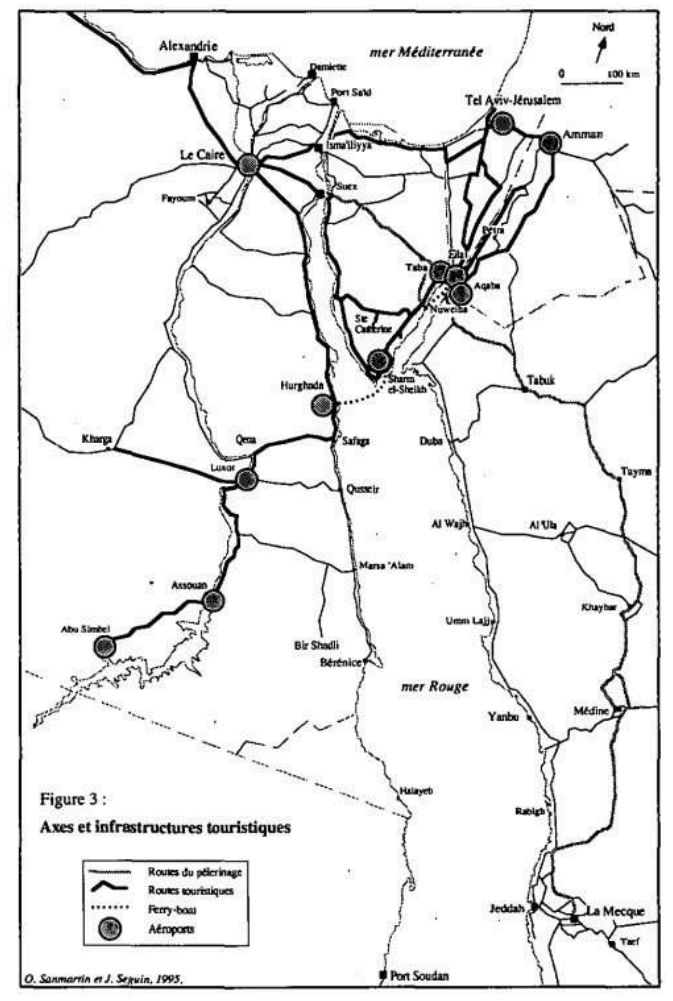

Désireuse de s'associer au projet, l'Égypte s'y intégrerait dans un premier temps par la mise en place d'infrastructures de transport qui relieraient les trois États. L'aéroport de Ra's al-Naqab, qui dessert actuellement Taba, devrait devenir «multinational». Un nouveau poste frontière, sur le plateau dominant le golfe, et une autoroute devraient connecter les trois pays sans entraves à la circulation, la définition d'une zone de libre échange permettant aux touristes de circuler sans visa ${ }^{54}$. Par ailleurs, l'Égypte a planifié de nouveaux complexes touristiques intégrés et ambitionne de créer autour de Taba, au 
nord du golfe, le pendant de ce que Sharm al-Shaykh est déjà au sud, à quelque 250 kilomètres. Il n'y a plus qu'à attendre les investisseurs...

51 Les interprétations divergent autour de ce projet, perçu tour à tour comme un espace touristique off-shore ou comme un premier signe de l'intégration régionale, à l'image des NET [Natural Economic Territories définis par le PNUD), ces «entités économiques franchissant les frontières politiques et combinant ressources, main-d'œuvre, capitaux, technologie et savoir de gestion $»^{55}$. La définition d'un système régional, la création d'un «centre" international et touristique dont le pôle majeur serait israélien suscitent néanmoins de nombreuses craintes, notamment côté jordanien. Avec 455.000 visiteurs en $1992^{56}-2,5$ millions en Égypte ${ }^{57}$ et 2 millions en Israël en $1993^{58}-$, le royaume hachémite, qui compte dépasser le million de touristes d'ici 5 ans $^{59}$, est pour l'heure sous-équipé et redoute la concurrence de ses voisins, qui le confinerait dans un rôle d'appoint, simple but d'excursion. Mais la mise en place de circuits touristiques à vocation régionale suscite également l'enthousiasme. Ainsi le responsable égyptien du comité du tourisme de l'Association des hommes d'affaires égyptiens imagine-t-il « un circuit qui commencerait à Louxor, se poursuivrait dans le Sinaï puis prendrait la direction de Pétra avant de se terminer à Jérusalem $»^{60}$. (Cf. figure 3)

$C^{\prime}$ 'est donc dans les relations que cet espace (essentiellement destiné à une clientèle occidentale) entretient avec le patrimoine touristico-culturel de l'ensemble régional que les acteurs économiques et politiques s'inscrivent. Mais dans un ensemble arabe où « il y a très peu d'échanges de biens et beaucoup de mouvements de personnes " ${ }^{61}$, il s'agit peut-être davantage de tirer profit d'une rente de situation que de faire sauter un verrou qui pourrait ouvrir la porte aux échanges entre le Golfe, l'Afrique du Nord et le Levant. Ne peut-on y déceler, au cœur d'une région où la question centrale - le devenir des Palestiniens - reste en suspens, une nouvelle forme de ségrégation spatiale? Les projets touristiques transfrontaliers marqueront-ils une rupture ou perpétueront-ils le mode de gestion de l'espace, et de ses ressources que pratiquent déjà individuellement les États? L'activité touristique est-elle apte à conduire un mouvement d'intégration, national ou régional?

Paysage et situation, de nouvelles rentes?

53 L'introduction puis le développement de la fonction touristique, l'appropriation du sol le long des littoraux par l'économie et la gestion de l'espace des loisirs internationaux ont-ils modifié la façon dont l'espace côtier était utilisé ? En d'autres termes, les activités qui s'y sont déployées réorientent-elles de façon irréversible les rapports qu'entretiennent ces espaces avec le territoire égyptien, avec l'économie égyptienne? N'assiste-t-on pas en réalité à la pérennisation d'une forme d'exploitation de l'espace côtier désertique, à la mise en place d'une nouvelle forme d'un même usage, le prélèvement d'une ressource et de la rente qui s'y rattache ? Le tourisme international s'apparenterait ainsi à une activité d'usage de la rente spatiale, de la ressource paysagère, elle aussi altérable, sinon épuisable.

La captation des rentes

54 Quel pourrait être le point commun entre l'aide étrangère, un bassin d'emploi, un site touristique et une plate-forme pétrolière? Rien, sinon le lien qui unit chacun de ces éléments à un "gisement» sur lequel on prélève une part importante du "prix de vente » de la ressource (au-delà des coûts de son exploitation) - ce que l'on pourrait appeler une rente - et qui se traduit par des flux importants de capitaux en devises. L'intérêt ici n'est pas de dresser un bilan de la rente, mais de mettre l'accent sur la 
relation qui s'instaure entre un espace et des flux. A ce titre, l'activité touristique centrée sur la mise en exploitation d'une ressource naturelle comme le paysage renvoie bien à cette catégorie. Les cinq types de rentes ${ }^{62}$ identifiés dans l'économie égyptienne sont liés à des gisements situés autour de la mer Rouge.

L'Égypte prélève sur son sol, comme d'autres pays de la région, une rente pétrolière. Si une part de la production (d'un total d'environ 900.000 barils par jour) revient aux compagnies étrangères qui exploitent les gisements et qu'une autre part est destinée à la consommation locale, le gouvernement égyptien perçoit bien le fruit de l'exportation d'environ $300.000 \mathrm{~b} / \mathrm{j}$.

La situation, définie comme le caractère d'un lieu "au sein d'un champ de forces, quelque part dans la structure du monde $»^{63}$, procure à l'Égypte trois autres types de revenus. Ceux liés au contrôle du passage à travers l'isthme de Suez, terrestre (oléoducs) ou maritime ; ceux liés à l'aide internationale récompensant des " choix " géopolitiques, en particulier depuis les accords de Camp David mais plus encore depuis la participation égyptienne à la guerre du Golfe, au côté des forces alliées. Enfin, le prélèvement opéré par la main-d'œuvre égyptienne dans les pays du Golfe, où la différence entre le coût de son "entretien », de sa "reproduction » et le revenu perçu permet le recyclage d'une partie de la rente des États pétroliers à travers des rapatriements de salaires vers l'Égypte.

La situation est par définition changeante, elle s'exploite tel un gisement, le maintien de son importance stratégique permet d'y prélever des ressources : des aides militaires et des allégements de dette, des salaires, des droits de passage. Mais ces ressources sont épuisables : réservoirs pétroliers, bassins de main-d'œuvre où se concurrencent des nationaux à forte natalité et des expatriés, trajectoires trans-isthmiques multipliées par l'intégration régionale d'Israël, qui pourraient, entre autres, porter préjudice au canal de Suez.

Tableau 4 : Evolution des rentes égyptiennes

\begin{tabular}{|c|c|c|}
\hline Millions \$ & 1991/92 & $1992 / 93$ \\
\hline 1 - Exportations de pétrole & $1.651,1$ & $1.802,9$ \\
\hline$\%$ des recettes d'exportations & 45,5 & 52,7 \\
\hline 2 - Recettes du canal de Suez & $1.950,2$ & $1.941,1$ \\
\hline$\%$ des recettes de services & 22,6 & 21,2 \\
\hline 3 - Recettes du tourisme \% des recettes de services & $\begin{array}{l}1.717,2 \\
20\end{array}$ & $\begin{array}{l}1.771 \\
19,3\end{array}$ \\
\hline $\begin{array}{l}\text { Recettes pétrolières, du canal et du tourisme en \% des revenus d'exportations } \\
\text { de biens et services }\end{array}$ & 43,4 & 43,8 \\
\hline 4 - Remise des travailleurs égyptiens expatriés & $5.477,9$ & $7.260,3$ \\
\hline 5 - Transferts publics sans contrepartie & $1.039,3$ & 1.357 \\
\hline
\end{tabular}




\begin{tabular}{|l||l|l|}
\hline Part des cinq postes cités dans les rentrées de capitaux à court terme en Égypte (\%) & 63 & 66,6 \\
\hline \hline Rappel : balance des paiements courants & $3.737,2$ & $4.773,3$ \\
\hline \hline Rappel : Solde commercial & - & $-7,314.5$ \\
\hline
\end{tabular}

Source : chiffres bruts de la Banque centrale et calculs des auteurs.

Les données financières publiées par la Banque centrale diffèrent de façon sensible de la comptabilité fournie par le Ministère du Tourisme, qui donne pour 1991-92 des recettes de 3 milliards \$. Le Ministère établit ses estimations en fonction d'une dépense moyenne par touriste et par jour, tandis que la Banque centrale enregistre des flux réels de capitaux à partir des données du système bancaire.

Le tourisme, d'abord basé sur une rente « historique » et désormais lié à l'exploitation d'une rente "paysagère ", n'échappe pas à ce type de contrainte : les paysages, les fonds marins sont des ressources fragiles qui peuvent ne pas résister à un usage intensif. La destruction des platiers et des récifs est causée aussi bien par la construction de pontons et de plages artificielles que par l'ancrage, la «cueillette » des coraux ou l'augmentation du nombre de plongeurs, qui ont déjà altéré les " gisements " des littoraux de Hurghada ou de Sharm al-Shaykh. Un «front de colonisation touristique » repousse sans cesse la limite de milieux naturels jusqu'alors préservés : vers le large, en direction des îles, d'une part, vers le sud de la côte orientale du gouvernorat de la mer Rouge, d'autre part ${ }^{64}$, où une politique d'aménagement tente de préserver des zones « tampons » face à l'avancée rapide des constructions et du mitage.

Les liens avec l'hinterland et l'avant-pays

Dans leur rapport à l'espace, les rentes de l'Égypte s'organisent bien plus en relation avec l'avant-pays ${ }^{65}$ qu'avec l'hinterland (l'arrière-pays). Les synapses qui mettent en contact les deux territoires du gisement et du "marché » en sont les ports et les aéroports. L'interface avec l'espace égyptien en est réduite à la trame encore lâche des réseaux routiers.

Le pétrole qu'extraient les compagnies étrangères et l'Egyptian General Petroleum Company (EGPC) à partir d'installations off-shore et terrestres est acheminé vers le nord des gouvernorats du Sud-Sinaï et de la mer Rouge, au débouché méridional du canal de Suez. L'avant-pays est en partie en Égypte, lorsque les hydrocarbures sont traités par les raffineries domestiques et distribués dans les réseaux, mais plus encore sur les marchés de consommation européens. Le phosphate prélevé dans le désert oriental, et bientôt au-delà du Nil vers l'ouest, avec l'ouverture de la voie ferrée du gisement d'Abû Tartûr, ne fait que traverser le territoire égyptien avant d'être exporté de Safaga, d'Hamrawayn ou de Qusayr vers des marchés lointains, notamment le marché australien.

61 Le tourisme des littoraux de la mer Rouge relève pour une part de ce type d'organisation. Des sites autonomes sont reliés aux pays exportateurs d'une clientèle touristique avec lesquels, par exemple, une amélioration «sélective» du réseau de télécommunication permet un contact direct ${ }^{66}$. La rente est prélevée, sur place par les propriétaires et les exploitants et rapatriée soit à l'étranger, soit hors du gouvernorat. Les retombées locales sont rares dans la mesure où une grande partie de la maind'œuvre des établissements touristiques est « importée » des gouvernorats de la vallée 
ou du Caire. Les populations locales, qui ne furent jamais associées à l'exploitation des ressources de leurs territoires, restent pour la plupart en marge de l'économie touristique, au sein de laquelle elles font figure d'élément folklorique, complément de la ressource paysagère. Les Bédouins dans le Sinaï, les artisans des chantiers de construction navale à Hurghada, ne prélèvent localement qu'une faible part des revenus générés par le tourisme.

Le changement d'échelle permet de comparer les configurations de prélèvement des ressources. Dans le cas des ressources minières, le gisement est éloigné du littoral, où s'opère la jonction de l'interface terrestre-maritime. Les réseaux d'oléoducs et de voies ferrées assurent cette liaison. Pour l'activité touristique internationale, la synapse et le gisement se confondent, l'espace intégré offrant l'infrastructure d'accueil (les aéroports) et la ressource. Cette configuration renforce d'une certaine façon - même si un réseau intérieur assure aussi la gestion des flux domestiques - l'autonomisation de cet espace de loisirs.

Logiques d'acteurs

63 Cette autonomie ne signifie pourtant pas une totale liberté d'action, car l'espace de prélèvement des rentes offre en Égypte la particularité d'être pluri-sectoriel. Aussi une compétition prend-elle forme pour l'appropriation du sol, entre les gestionnaires de l'environnement stratégique, les militaires, ceux du territoire minier et les acteurs $\mathrm{du}$ développement touristique. Quelques exemples permettent d'en juger : en mai $1994^{67}$, des investisseurs égyptiens (dont Muhammad Faris, ainsi que Sa'îd al-Tawwîl, président de l'Egyptian Businessmen's Association) ont présenté devant la Haute Cour du Caire une requête contre la décision de l'Autorité pour les investissements de retirer la licence d'exploitation de la Sahl Hashish Bay Development Company, société financière maître d'ouvrage d'un complexe touristique au nord de Safaga, pour cause de retard dans l'exécution du projet. Or, il est apparu que le préjudice était en réalité causé par les délais imposés par le Ministère de la Défense pour la mise à disposition du terrain. Les militaires conservent en effet des moyens de pression et des relais au sein des administrations. Ce sont d'anciens officiers qui occupent les plus hautes fonctions administratives au sein des gouvernorats : gouverneurs, directeurs exécutifs...

En 1993, en affirmant la «vocation » essentiellement touristique du Sud-Sinaï, c'est le gouverneur qui a tranché, officiellement, le différend qui opposait les Ministères du Pétrole et du Tourisme, ce dernier entendant freiner la progression de l'exploitation pétrolière vers le sud de la côte occidentale du Sinaï au profit du développement touristique. De même, dans le gouvernorat de la mer Rouge, les Ministères du Pétrole et du Tourisme ont dû régler par un accord, signé en mars $1992^{68}$, un contentieux portant sur la délimitation de leurs zones d'intervention. L'activité de prospection pétrolière et le développement des loisirs aquatiques s'avérant incompatibles, un arrangement a été dégagé, permettant de préserver des sites exclusivement consacrés au tourisme (péninsule de Ra's Banas, littoral de Hurghada à Safaga, mais pas l'hinterland proche), et des sites communs (région de Qusayr, sud de Marsa Alam) où les intérêts des deux parties doivent être préservés. Les conflits portant sur la propriété du sol et les attributions de chacun sont légion. Les négociations ont été serrées, en particulier entre la TDA (Tourism Development Authority), qui possède les terrains en dehors des limites des municipalités, et ces dernières, qui trouvent intérêt à étendre leurs circonscriptions, en particulier sur l'espace côtier ${ }^{69}$. A Marsa Alam, un différend a 
opposé les Ministères du Tourisme et de la Reconstruction à propos de la gestion des terrains ${ }^{70}$.

Au-delà des ministères, soucieux de leurs prérogatives, les entrepreneurs, nous l'avons $\mathrm{vu}$, occupent désormais une place prépondérante dans la gestion de l'espace et dans le processus de décision. Si la loi 230 de 1989 a élargi les privilèges des investisseurs par rapport aux mesures de l'infitâh, les participations étrangères restent limitées à la constitution de joint-ventures à majorité égyptienne, particulièrement dans le Sinaï où la possession d'actifs par des étrangers est, en principe, proscrite. L'ouverture, à la lin de 1994, d'un complexe touristique (100 millions \$ d'investissement) dont la société, maître d'ouvrage et propriétaire du terrain, est détenue à $75 \%$ par un homme d'affaires italien ${ }^{71}$, constitue la première dérogation à la loi. Signe d'un changement notable dans la gestion d'un espace "sensible", sur lequel de nombreux acteurs entendent conserver un "droit de regard», ce précédent pourrait bien être suivi d'autres initiatives du même genre. Les messages rassurants et les appels répétés des dirigeants pour drainer les capitaux égyptiens expatriés ${ }^{72}$ ayant un impact limité, le capital étranger devrait se trouver de plus en plus sollicité.

Enfin, les organismes chargés de la protection de l'environnement, omniprésents depuis la conférence de Rio, constituent désormais un groupe de pression apte à imposer des mesures de sauvegarde et des normes, notamment en matière de construction, dans un domaine où le vide juridique ne permettait pas un contrôle efficace. Relayées par les pouvoirs locaux, notamment dans le Sinaï, les préoccupations «environnementales " participent d'une prise de conscience plus large qui associe préservation du milieu et performances économiques, dans une région qui abrite le premier Parc national d'Égypte ${ }^{73}$. La prise en compte de mesures de protection de l'environnement est désormais une condition sine qua non pour l'obtention de prêtsprojets attribués par les bailleurs de fonds multilatéraux ou bilatéraux.

Vers la complexification, l'intégration?

Les problèmes d'arbitrage entre les responsables d'activités sinon antagonistes, du moins concurrentes, pour l'appropriation des territoires révèlent d'une certaine façon le dynamisme des espaces côtiers et la multiplicité des projections planificatrices qui s'y rattachent. Si le partage des zones d'intervention est plus délicat dans le désert oriental que dans le Sud-Sinaï, les deux gouvernorats font chacun l'objet d'une planification à long terme destinée à mobiliser l'ensemble des ressources disponibles et à densifier les réseaux qui doivent les connecter au reste du territoire égyptien. A l'instar des gouvernorats de la vallée, qui se sont agrandis vers l'est en intégrant pratiquement la moitié de la superficie initiale du gouvernorat de la mer Rouge, les littoraux sont amenés à s'ouvrir aussi à l'espace intérieur. Le développement du tourisme domestique, déjà important sur le littoral méditerranéen, bien au-delà d'Alexandrie, encore marginal dans ces régions éloignées, en est peut-être un des signes précurseurs.

C'est sans doute grâce à une plus grande intégration des économies locales à l'armature nationale, grâce à la densification des connexions tirées à partir de la vallée, que peuvent être dépassées les contradictions. Si les premières planifications territoriales des gouvernorats se sont inspirées un temps de la spécialisation fonctionnelle des espaces $^{74}$, les nécessités de l'intégration ont amené les planificateurs à modifier cette organisation. Le plan de développement en cours d'achèvement par l'équipe d'experts des Nations Unies au sein du Ministère de la Planification, plan qui couvre l'ensemble 
du sud de l'Égypte, de la vallée au littoral, témoigne d'une volonté de concevoir l'aménagement du territoire d'une manière plus intégrée. Dans une même logique, un développement pluri-sectoriel ambitieux de la péninsule du Sinaï est projeté sur 22 ans $^{75}$.

Pourtant, le rythme de la croissance économique et spatiale des littoraux semble encore en décider autrement. Le décalage entre le temps de la planification et le temps opérationnel est une des données explicatives du retard pris dans la densification de l'armature transverse, tandis que les réponses institutionnelles à la $\mathrm{crise}^{76}$, en réorientant les priorités, se traduisent par des mises en chantier permanentes dont les aboutissements sont bien souvent aléatoires. Terrains d'expérimentation, d'évolution, lieux de production spatiale et donc de production sociale, les littoraux de la mer Rouge, en se complexifiant, sont peut-être en passe de devenir des espaces importants dans la genèse de nouvelles pratiques et de nouvelles perceptions du territoire, affranchis des contraintes qui ont longtemps façonné l'espace égyptien.

Figure 4 : Les littoraux égyptiens de la mer Rouge : essai de modélisation

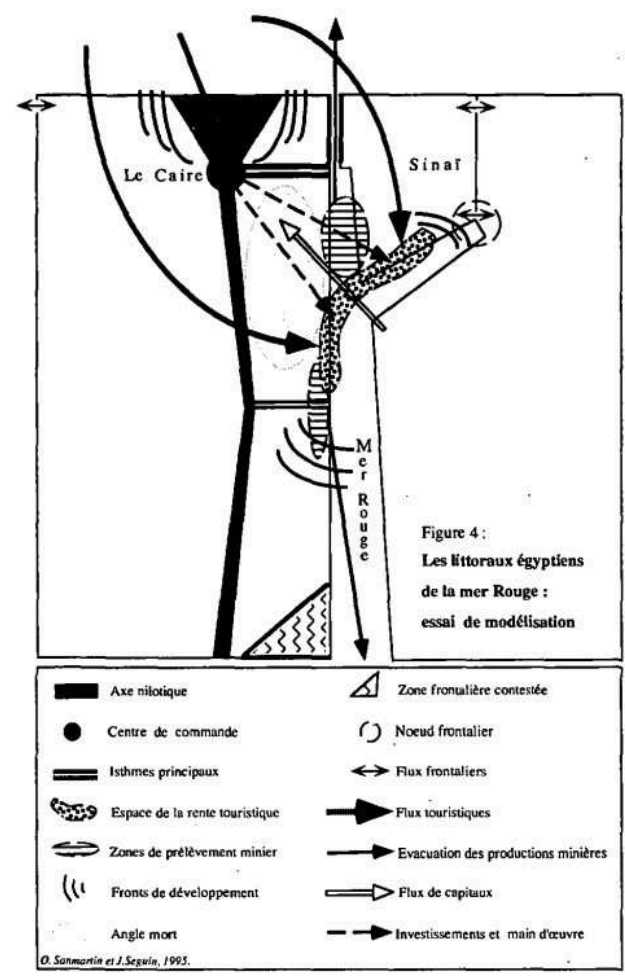

Si l'autonomisation des espaces voués à la fonction touristique en limite nécessairement l'impact sur le développement national, les dynamiques locales semblent néanmoins porteuses d'un changement social qui reste encore difficile à cerner. Les flux migratoires à destination des régions touristiques, à la différence des centres urbains ou industriels, sont essentiellement masculins, connaissent des irrégularités saisonnières voire interannuelles et sont généralement peu "intégrateurs ${ }^{77}$. Pourtant, dans un pays où l'on se déplace beaucoup, où les perspectives de migration dans le Golfe se tarissent, où le secteur public n'est plus à même d'assurer la promotion sociale d'une jeunesse nombreuse, on peut formuler l'hypothèse que la perception, par les Égyptiens, d'une installation dans les marges 
désertiques du pays, traditionnelles zones d'aversion, est en train de changer ${ }^{78}$. (Cf. figure 4)

71 Intégration, extraversion, reconnexion : les espaces touristiques des littoraux de la mer Rouge relèvent d'une certaine façon de lieux communs... Le désenclavement, par le tourisme, "d'isolats» ou "d'angles morts " $^{79}$, les redistributions vers des espaces marginaux riches en ressources touristiques, sont des phénomènes classiques, maintes fois relevés par les études concernant le développement touristique et particulièrement dans les pays du tiers monde ${ }^{80}$. Ce qui est peut-être plus spécifique, c'est, d'une part, que ces processus prennent place sur des espaces ayant une forte charge symbolique, notamment dans le rôle qu'ils jouent pour assurer la sécurité de la nation - ils se situent à un nœud isthmique régional, une interface « dangereuse »-, et, d'autre part, que l'émergence de ces nouveaux territoires répond à la volonté, sans cesse réaffirmée, d'une redistribution du peuplement, enjeu de politiques et de discours mobilisateurs visant à libérer l'espace nilotique d'une pression démographique qui mettrait en cause le développement national.

En marge du territoire, les littoraux sont les vecteurs d'une dynamique centripète qui esquisse une nouvelle organisation de l'espace national. Parce que la «marge » de manœuvre y est plus grande, des productions de l'espace s'y déploient qui renouvellent la relation des Égyptiens à leur territoire.

\section{ANNEXES}

Évolution du nombre de touristes en Égypte entre 1966 et 1994

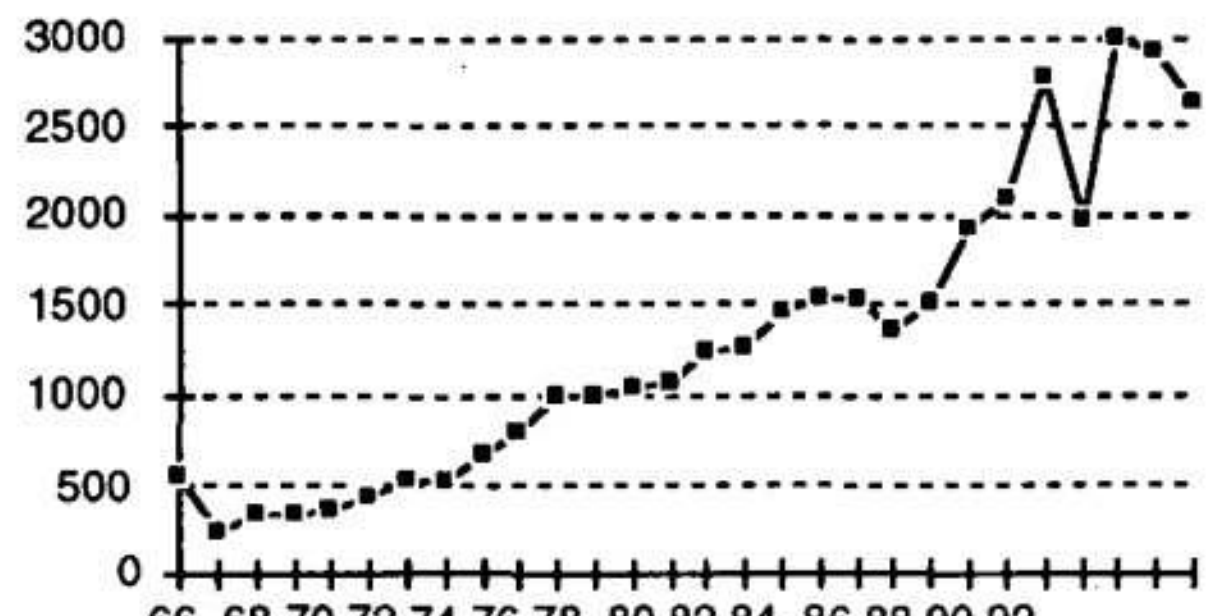

$66 \quad 68707274767880828486889092$

Sources : annuaires statistiques de la Capmas de 1970 à 1994 (en année fiscale à partir de 1980, ainsi le dernier chiffre correspondant à 1993-94).

Arrivées à l'aéroport de Hurghada en 1989 


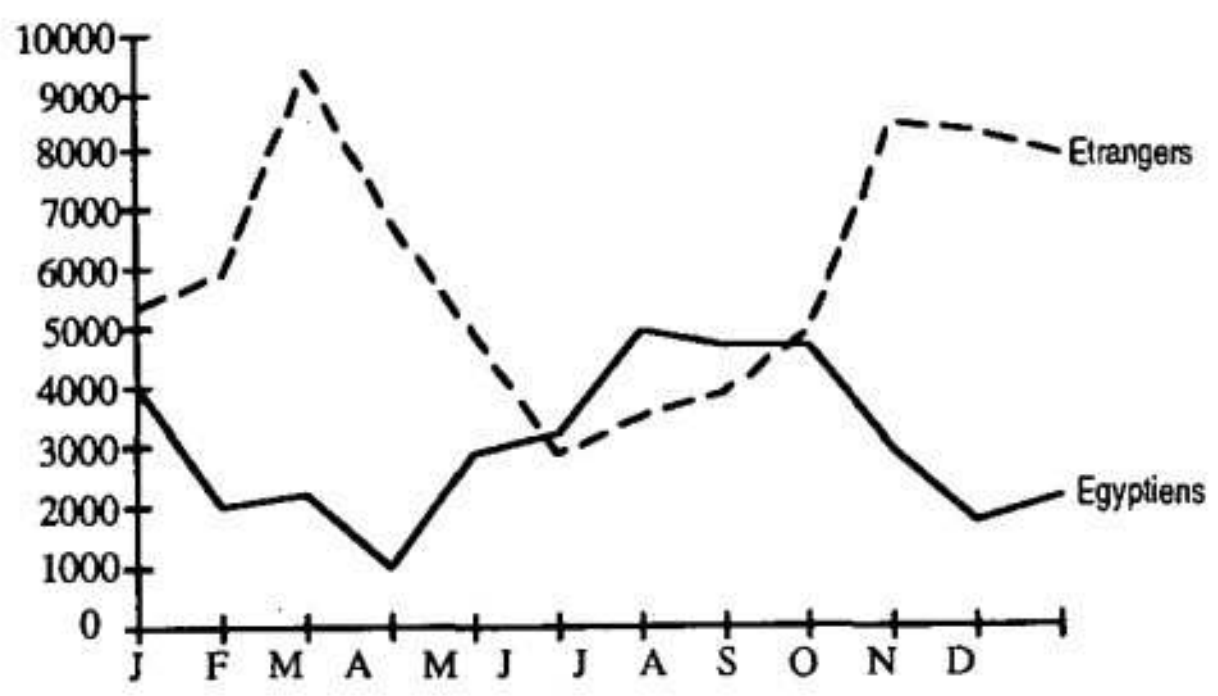

Source : GOPP, juin 1993.

\section{NOTES}

1. La notion d'échelle est ici entendue au sens géographique du terme, "petite échelle » faisant référence à une grande portion d'espace et inversement.

2. Estimation pour juillet 1993, basée sur les chiffres du recensement de 1986 et l'accroissement naturel. Ce chiffre peut être réévalué puisqu'il ne tient pas compte des mouvements migratoires. Capmas, Statistical Year Book, juin 1994.

3. Abd Al-Maqsud A. M., « La politique des villes nouvelles en Égypte : tentative d'évaluation du point de vue spatial », Dossier du Cedej 2/1987, pp. 31-51.

4. Brunet R., Géographie universelle, t.1 : Mondes nouveaux, Hachette- Reclus, Paris, 1990, $552 \mathrm{p}$.

5. Voir les remarques de Gamâl Hamdan sur cette notion dans son ouvrage, Shakhsyat misr ; dirâsât fi jughrafiyya al-makân, vol. 1, 'Alam al-kutub, Le Caire, 1980, 841 p.

6. Métaphore proposée par R. Brunet, op. cit.

7. Cité par D'Istria P., De Suez à Akaba, Cujas, Paris, 1969, 160 p.

8. Wissa K., «The Oil Question in Egyptian-Israeli Relations, 1967-1979 : A Study in International Law and Resource Politics ", Cairo Papers in Social Science, AUC Press, Le Caire, 1989, vol. 12, n 4, 116 p.

9. Hobbs J. J., Beduin Life in the Egyptian Wilderness, AUC Press, Le Caire. 1990, 165 p.

10. Taba, à la frontière égypto-israélienne, est un territoire de $1 \mathrm{~km}^{2}$ qu'Israël refusa de restituer à l'Égypte lors du retrait du Sinaï en 1982. Un arbitrage international statua en faveur de l'Égypte et l'enclave fut évacuée en mars 1989.

11. Garcin J.-C., Un centre musulman de la Haute-Égypte médiévale : Qus, IFAO, Le Caire, $1976,658 \mathrm{p}$.

12. Sayyed A. M., Al-bahr al-ahmar wa dhahîruhu fi-l-'usûr al-qâdîma, Dâr al-ma'rifa algamî'a, Le Caire, 1993, 831 p.

13. Weill R., La presqu'île du Sinaï. Etude de géographie et d'histoire, Librairie Honoré Champion, Paris, 1908, 380 p. 
14. Tregenza L. A., The Red Sea Mountains of Egypt, Oxford University Press, Londres, 1955,247 p.

15. Alleaume G., « La naissance de la géographie positive et les Khitat-s de 'Alî pacha Mubârak ", D'un Orient à l'autre, vol, 2, Presses du CNRS-Cedej, Paris, 1991, pp. 315-328. 16. Sidqi Pacha S.E.I., « Les mines d'Égypte », Bulletin de la Société Sultanienne de Géographie d'Égypte, vol 2, Le Caire, 1922, pp.148-170.

17. Habashi F. et Bassyuni F A., Mineral Ressources of the Arab Countries, Chemecon Publishing, Londres, 1982, $60 \mathrm{p}$.

18. Sidqi, op. cit.

19. Loi du 18/01/1951, amendée par décret du 17/02/1958 (cf. WISSA, op. cit).

20. Ibid.

21. Al-Gamasy M., The October War, AUC Press, Le Caire, 1993, 438 p.

22. Al-Ahrâm, 12/01/1994.

23. Cette base, située au sud du gouvernorat de la mer Rouge, a été l'objet d'une controverse entre le gouvernement égyptien et l'État-major américain : ce dernier souhaitait l'utiliser et la développer dans le cadre de sa Force de déploiement rapide. Les Égyptiens ont refusé ce qu'ils considéraient comme une ingérence caractérisée, mais la flotte américaine n'en continue pas moins d'y disposer de « facilités ».

24. Le Progrès Égyptien, 22/10/1993.

25. Middle East Times, 8-14/01/1995.

26. Editorialiste d'al-Sha'b, organe du Parti du Travail, d'obédience islamiste.

27. Al-Sha'b, 23/12/1994.

28. Middle East Times, op. cit.

29. Haaretz, cité par l'AFP, 18/11/1992.

30. Un plan de développement (1992-1997) prévoit, outre la sédentarisation des populations locales, l'implantation de 6.000 Égyptiens, selon al-Wasat, 29/11/1993.

31. La création du Condominium anglo-égyptien sur le Soudan en 1899 fut à l'origine du tracé d'une frontière administrative entre l'Égypte et le Soudan, le long du 22e parallèle, alors que l'ancienne limite septentrionale du Soudan égyptien (1882) remontait plus au nord, jusqu'au $24^{\mathrm{e}}$ parallèle, aux environs de Bérénice. La zone comprise entre les deux lignes, administrée par le Soudan depuis son indépendance en 1956, est l'objet d'un litige sur lequel les interprétations juridiques divergent. Les Égyptiens souhaitent un arbitrage international, mais les discussions sont actuellement au point mort et l'Égypte occupe de fait le secteur, alors que le Soudan conditionne la normalisation de ses relations avec l'Égypte au retrait du triangle d'Halaïb et à l'expulsion des opposants soudanais.

32. «Chronologie politique ", 24/11/1992, Égypte/Monde arabe n¹2-13, $4^{\mathrm{e}}$ trim. 1992 et $1^{\mathrm{er}}$ trim. 1993, Cedej, p. 334.

33. Traité de non-prolifération nucléaire, dont l'Égypte est signataire.

34. Tourism Development Authority (1992) : Egypt's Tourism Development Program and Investment Opportunities, Ministère du Tourisme, Le Caire, $56 \mathrm{p}$.

35. Le Progrès Égyptien, 22/12 et 19/11/1994.

36. Jobbins J., The Red Sea Coasts of Egypt : Sinai and the Mainland, AUC Press, Le Caire, $1989,116 \mathrm{p}$.

37. Situé sur la côte du gouvernorat de la mer Rouge, au nord de Safaga.

38. AI-Ahram Hebdo, 18-24/01/1995.

39. Le recensement de 1976 ne comprenant pas le Sinaï, alors occupé, un recensement a été entrepris en 1982, après la restitution de la péninsule à l'Égypte. 
40. SATEC et al., Red Sea Governorate Regional Plan, Interim Report : Tourism, Le Caire, 1980, $39 \mathrm{p}$.

41. CAPMAS, Al-sukkân wa ahamm al-anshita al-sukkâniya, muhâfaza al-bahr al-ahmar, Le Caire, 1992, 76 p.

42. Al-Ahrâm, 12/02/1995.

43. Al-Hayat, 3/12/1994.

44. CAPMAS, Statistical Yearbook, 1994 ; en comparaison, les aéroports du Caire et de Louxor ont accueilli respectivement 6.738 .000 et 1.989 .000 passagers.

45. Al-Ahram Hebdo, 12-18/10/1994.

46. Le Progrès Égyptien, 04/12/1994. Depuis la fin de 1994, quatre banques d'État égyptiennes sont autorisées à échanger la monnaie israélienne : la Banque Misr, al-Ahli, la Banque du Caire et la Banque d'Alexandrie. Ces banques font ensuite le change contre des dollars avec la banque israélienne Leumi.

47. General Organization for Physical Planning, Al-tanmiyya al-'umrâniyya al-shâmila li-liqlîm al-bahr al-ahmar : al mukhattat al-'âmm li-madinat al-ghardaqa, 2020, Le Caire, 1993, $140 \mathrm{p}$.

48. Brunet R., Les mots de la géographie, Reclus-La Documentation française, Paris, 1992, $470 \mathrm{p}$.

49. Décret présidentiel $n^{\circ} 24 / 1994$, Journal Officiel $n^{\circ} 5,3 / 02 / 94$

50. Brunet R., op. cit.

51. A cet égard, l'ouverture, en 1994, d'une ligne maritime directe entre les ports de Safaga et de Duba sur la côte saoudienne, est significative de cette évolution.

52. Business Week, 23/05/1994.

53. « The Eilat Free Trade Zone », The Israeli Economist n 5, mai/Juin 1989.

54. AI-Wasat, 7/11/1994.

55. Decornoy J., « Délicate fin de guerre dans la péninsule de Corée », Le Monde Diplomatique, novembre 1994, pp. 22-23, citant Scalapino R., « The Majors Powers and Korean Peninsula », in Korean Journal of National Unification, 1994.

56. Ministère du Tourisme, Tourism in Figures, Le Caire, 1993, 152 p.

57. Ibid.

58. Keath L, « The Peace Road », Egypt Today, janvier 1995, pp. 92-98.

59. Al-Hayat, 12/11/1994.

60. Keath L., op. cit.

61. Cabrillac B., «Les spécificités de l'économie égyptienne », Égypte/Monde arabe $\mathrm{n}^{\circ}$

12-13, $4^{\text {e }}$ trim.1992 et $1^{\text {er }}$ trim.1993, Cedej, pp. 11-19.

62. Ibid.

63. Brunet R., op. cit.

64. Gulens Mara, « The Last Frontier », Egypt Today, vol. 16, n² 2, 1995, pp. 96-99.

65. Avant-pays : espace apportant les entrées dans un port maritime ou recevant les sorties. L'avant-pays est donc maritime et continental (d'outre-mer).

66. Ayeb H., « Infrastructures et réseaux hydrauliques : une nouvelle carte de l'Égypte ", Égypte/Monde arabe n¹, 1990, pp. 11-23.

67. Middle East Economic Digest, 6/05/1994.

68. Ministère du Tourisme, document de six pages avec une carte en annexe.

69. Entretien avec 'Adil Radi, ingénieur à la TDA.

70. Akhbâr al-Yawm, 04/09/1993.

71. «Chronique économique ", Égypte/Monde arabe $\mathrm{n}^{\circ} 15-16,3^{\mathrm{e}}$ et $4^{\mathrm{e}}$ trim. 1993 , Cedej, p. 510. 
72. Selon un document de travail de la Banque mondiale, l'épargne privée détenue à l'étranger par des résidents égyptiens s'élevait à 82,6 milliards \$ en 1991 (cf.

«Chronique économique ", Égypte/Monde arabe n¹7, 1er trim. 1994, Cedej, p. 233). Ce

chiffre extrêmement élevé peut être corrigé par une autre évaluation, celle des revenus d'investissement à l'étranger, qui se monteraient à 1,5 milliard \$ en 1991-92. Cf.

Cabrillac B., op. cit.

73. II s'agit du Parc national de Ra's Muhammad, à l'extrême sud de la péninsule.

74. Le gouvernorat de la mer Rouge a fait par exemple l'objet d'un découpage en « lanières » longitudinales, chacune étant affectée à une spécialisation sectorielle : agriculture près de la vallée, mines à l'intérieur et tourisme côtier.

75. Un plan qui devrait bénéficier de 75 milliards LE d'investissements, approuvé en octobre 1994, prévoit le peuplement du Sinaï par 3 millions de personnes, la création de 500.000 emplois et la bonification de 400.000 feddans d'ici 2017.

76. La priorité désormais énoncée est le développement du Sa'îd (le sud de l'Égypte), foyer de la contestation et région décrite comme la plus sous-développée du pays. De nouvelles mesures destinées à attirer les investissements (projets industriels notamment) ont été prises récemment par le gouvernement.

77. Cazes G., Tourisme et tiers-monde, un bilan controversé. Les nouvelles colonies de vacances?, L'Harmattan, Paris, 1992, $207 \mathrm{p}$.

78. Nous avons entamé une enquête dans ce sens auprès d'un échantillon de population, essentiellement cairote, et les premiers résultats semblent confirmer cette hypothèse.

79. Reynaud A., Société, espace et justice, PUF, Paris, 1981, 263 p.

80. Cazes G., op.cit.

INDEX

Mots-clés : géographie, économie, Sinaï, tourisme, sécurité

\section{AUTEURS}

OLIVIER SANMARTIN

CEDEJ

JACQUES SEGUIN

CEDEJ 